\title{
The Effect of Flurbiprofen Lozenge on Pain, Oedema, and Trismus after Impacted Third Molar Surgery: A Prospective Randomized, Double-Blinded, Placebo-Controlled Study \\ Dr. Tejashree $\mathrm{R}^{*}$ and Dr. Hemalatha BR
}

Department of Dentistry, Shri Atal Bihari Vajapayee Medical College and Research Institute, Bangalore, Karnataka, India

DOI: $10.36347 /$ sjds.2021.v08i05.007

| Received: 13.05.2021 | Accepted: 17.06.2021 | Published: 23.06.2021

*Corresponding author: Dr. Tejashree $\mathrm{R}$

Abstract

Original Research Article

Background: The postoperative sequelae after third molar surgery include pain, swelling and trismus, Flurbiprofen is a non-steroidal anti-inflammatory drug (NSAID), and the anti-inflammatory mechanism of Flurbiprofen is thought to involve inhibition of prostaglandin biosynthesis, in common with other NSAIDs. Prostaglandins such as prostaglandin E2 (PGE2) and other inflammatory mediators released in response to pain or non-infectious stimulants trigger a complex inflammatory cascade that contributes to inflamation symptomsThis study was aimed to evaluate the efficacy of oral Flurbiprofen lozenge given $30 \mathrm{~min}$ before surgery on reducing postoperative sequelae. Method: We recruited 70 patients, randomly assigned to two groups: Flurbiprofen and Control group, groups received 100mg Flurbiprofen lozenge, placebo lozenge $30 \mathrm{~min}$ before surgery respectively and every $6 \mathrm{~h}$ till $72 \mathrm{~h}$ after surgery. Third molar extraction was performed under local anesthesia. After extraction, pain, swelling, and mouth opening in both groups observed till 72h. Statistical Analysis: Preoperative and postoperative measurement of visual analog scale scores for pain, edema, interincisal opening, was analyzed using Student $t$ test or ANOVA, Chi-square or Mann-Whitney $U$ test was performed for non-parametric samples. $\mathrm{P}<0.05$ was considered as statistically significant. Results: The overall incidence of Pain in the Flurbiprofen group was $18 \%$ compared to $43 \%$ in the Control group $(P=.003)$ Incidence of Pain, swelling, and mouth opening at different time intervals $6 \mathrm{~h}, 24 \mathrm{~h}, 48 \mathrm{~h}$ and $72 \mathrm{~h}$ were significantly $(P>0.05)$ better in Flurbiprofen group. Conclusion: Oral Flurbiprofen lozenge administered 30 minutes preoperatively can significantly reduce postoperative sequelae after third molar extraction.

Keywords: Postoperative sequelae, Third molar, Flurbiprofen, Placebo, Trismus.

Copyright $\odot 2021$ The Author(s): This is an open-access article distributed under the terms of the Creative Commons Attribution 4.0 International License (CC BY-NC 4.0) which permits unrestricted use, distribution, and reproduction in any medium for non-commercial use provided the original author and source are credited.

\section{INTRODUCTION}

Third molar extractions are one of the most common and basic outpatient interventions in oral and maxillofacial surgery [1]. Depending on the location, depth, tooth angulation and bone density, the complexity of surgical extraction may vary, and is generally associated with postoperative sequelae [2]. Recently some strategies have been developed for minimizing postoperative discomfort after third molar surgery, including the use of pharmacological therapy and alternative medicine [3], and complementary protocols like minimally-invasive tooth extraction have been suggested for the postsurgical therapy of third molar surgery [4]. However, patients still suffer some pain, swelling, and limitation in mouth mobility and other symptoms after surgery. Reducing dental malaise and postoperative complications is a critical issue for oral and maxillofacial surgery doctors [5].
Flurbiprofen is a non-steroidal antiinflammatory drug (NSAID), the anti-inflammatory mechanism of Flurbiprofen is thought to involve inhibition of prostaglandin biosynthesis, 24 in common with other NSAIDs. [6] Prostaglandins such as prostaglandin E2 (PGE2) and other inflammatory mediators released in response to pain or non-infectious stimulants trigger a complex inflammatory cascade that contributes to inflamation symptoms [7].

PGE2 may impact activation of the vanilloid receptor (TRPV1) in sensory neurones, which is associated with pain pathways [8]. Flurbiprofen locally administered as lozenge or spray, which provides rapid and long-lasting sore throat relief [9].

At present, the clinical evidence regarding the effect of Flurbiprofen is sparse. In light of these findings, this study was conducted to compare the 
Tejashree R \& Hemalatha BR., Sch J Dent Sci, Jun, 2021; 8(5): 160-165

effects of commercially available Flurbiprofencontaining lozenges given $30 \mathrm{~min}$ before surgery on reducing pain during 3 days after surgical extraction of mandibular third molars.

\section{MATERIALS AND METHODS}

This prospective randomized controlled trial was conducted during May 2019 and April 2020. It was reviewed and approved by the Institutional Ethics Committee; we recruited 70 patients after obtaining written informed consent, before the beginning of this study with mandibular-impacted third molar. Inclusion criteria: age between 18 and 30 years, American Society of Anesthesiology (ASA) score of 1 (i.e., no systemic diseases or medical conditions), no active pathology associated with the third molars, no acute pericoronitis, and no periodontal disease. Exclusion criteria: contraindications of surgery; long-term administration of medication, recent administration of steroids, oral and systemic antibiotics, or repeated pericoronitis of the tooth; caries of moderate degree of the wisdom and adjacent teeth; periapical periodontitis, pulpitis, or periodontitis, anti-platelet or anticoagulant therapy, pregnancy or lactating, recent local infection within 15 days prior to surgery.

Sample size calculated by analyzing previous studies, presuming the incidence of postoperative pain to be $65 \%$. Using power analysis, as per the sample-size calculation 31 patients per group would be required to detect a minimum of $50 \%$ reduction in the incidence of postoperative pain, with alpha $=0.05$ and $\beta=0.20$ for two-tailed statistical analysis. Therefore, we included thirty five patients in each group.

We divided patients equally and randomly into Flurbiprofen group and Control group, using a computer randomization generator, each with 35 patients. Perioperative treatment for patients in the two groups was conducted using the double-blind method. Before surgery all patients routinely examined and dental X-ray film was obtained before surgery. The extent of swelling, limitation of mouth opening index, and data related to procedure were measured before surgery.

All patients in the group received Lozenge 30 min before surgery, followed by every $6^{\text {th }}$ hour for $72 \mathrm{hrs}$.

1. Group F (Flurbiprofen group) $(n=35)$ - oral Flurbiprofen lozenge $(8.75 \mathrm{mg})$

2. Group $C$ (Control group) $(n=35)$ - oral lozenge as placebo.

Flurbiprofen lozenge $-8.75 \mathrm{mg}$ Flurbiprofen, sucrose, cellulose powder, xanthan gum, sodium cyclamate, aromatics, and colorant, Placebo lozengecontaining sucrose, sodium free, and calcium with xylitol, glucerin and which is indistinguishable in appearance and taste from the one containing Flurbiprofen lozenge. Lozenge will be provided by the one staff nurse and subsequent assessment of the patient by different staff nurse. Patients and operating surgeon will not be aware of the study drug.

All surgical procedures were performed by one oral and maxillofacial surgeon; aseptic scrubbing and draping were done. Under strict aseptic precautions, 2 $\mathrm{ml}$ of $2 \%$ lignocaine with $1: 2,00,000$ adrenaline was used for an inferior alveolar nerve block, following the hospital's protocol. Additionally, infiltration anesthesia was administered in the buccal fold and distal of the incision in the mandibular ramus region.

A standard Ward's incision or a modified Ward's incision was given regularly for all the cases. In cases where visible intraoral crown or part of crown of the tooth, a standard ward's incision was placed. In cases if tooth was completely covered by mucosa, modified ward's incision was placed. To eliminate bias, we used only modified ward's incision for both control and study side. To expose the tooth and surrounding bone a full- thickness mucoperiosteal flap was raised. We used a round bur and a straight fissure bur no 701, for guttering buccal bone. The arc of rotation was determined and analyzed using preoperative radiographs. If interference to removal was found on analysis, sectioning was done using a no 703 straight fissure bur. After extraction, granulation tissue, follicular remnants and bony spicules were removed from the socket and closure done in both groups with 3- 0 silk sutures. Patients were given both verbal and written postoperative instructions after surgery, Patients in both groups were provided with an ice pack for postoperative cooling, and were prescribed 3- day course of amoxicillin + clavulanate $1000 \mathrm{mg}$ - twice daily, all patients were given comprehensive instructions on the importance of maintenance of oral hygiene and jaw physiotherapy postoperatively.

Detection of indexes and evaluation. The visual analog score (VAS) was adopted to evaluate the pain degree of patients. The score range was $0-10$ ( 0 for no pain and 10 for intolerable severe pain) severe: $\geq 7$ points for intolerable pain, patients took a combination of ibuprofen $400 \mathrm{mg}+$ Paracetamol $325 \mathrm{mg} 1$ tablet, The patient marked the appropriate response on the scale at $24 \mathrm{~h}, 48 \mathrm{~h}$ and $72 \mathrm{~h}$ after surgery.

The measurements were obtained using a flexible ruler in the pre and post-operative periods of 6 , 24,48 and $72 \mathrm{~h}$. The sum of the pre-operative measurements was considered the standard of normality for each side. The measurements from the postoperative period was verified, the difference between before and after the surgical procedure measurements was observed, determining the level of oedema. 
Tejashree R \& Hemalatha BR., Sch J Dent Sci, Jun, 2021; 8(5): 160-165

Trismus was evaluated by measuring the maximum buccal opening. Using a digital caliper transcribed in millimeters, we measured the maximum inter-incisional vertical distance (distance between the surfaces of the upper and lower right central incisors) after maximum opening without aid, pre-operatively and $6 \mathrm{~h}, 24 \mathrm{~h}, 48 \mathrm{~h}$ and $72 \mathrm{~h}$ post-operatively. The measurements were compared to the baseline. Thus, the relative mean (Delta) was calculated for each patient between the final and initial measurements.

Statistical calculations were conducted using SPSS (Statistical Package for the Social Sciences) Version 24.0 (IBM Corporation, Chicago, USA). The parametric variables were presented as mean \pm SD and analyzed by Student $\mathrm{t}$ test or ANOVA and Pearson correlation test as appropriate, Chi-square or MannWhitney U test and Spearman correlation coefficients was performed for non-parametric samples. $\mathrm{P}<0.05$ was considered as statistically significant.

\section{RESULTS}

A total of 70 patients were included in the study and randomly assigned to one of the groups. Thirty five patients received Flurbiprofen and 35 received placebo lozenge. There were no significant differences between two groups of patients with respect to age, sex, and BMI (Table I).

Table-I: Demographic Characteristics of Patients in Two Groups
\begin{tabular}{|l|l|l|l|}
\hline Variables & $\begin{array}{l}\text { FLURBIPROFEN } \\
(\text { mean } \pm \text { SD) }\end{array}$ & $\begin{array}{l}\text { CONTROL } \\
(\text { mean } \pm \text { SD) }\end{array}$ & P Value \\
\hline Age (year) & $29.2 \pm 8.6$ & $27.4 \pm 10.8$ & 0.44 \\
\hline Male (\%) & 57.1 & 51.4 & 0.63 \\
\hline Weight (Kg) & $74.4 \pm 12.6$ & $75.2 \pm 12.8$ & 0.79 \\
\hline BMI (kg/m2) & $22.4 \pm 3.2$ & $23.1 \pm 4.2$ & 0.43 \\
\hline
\end{tabular}

The overall incidence of Pain in the Flurbiprofen group was $15 \%$ shown in green ( Fig -1) compared to $43 \%$ in the Control group shown in red (P $=.010$ ) using generalized estimating equation with binomial probability distribution, logit link function, and autoregressive (AR) working correlation matrix resultant in odds ratio (95\% confidence interval), 6.568 (6.711 to 46.758 ). Error bars represent $95 \%$ confidence interval.

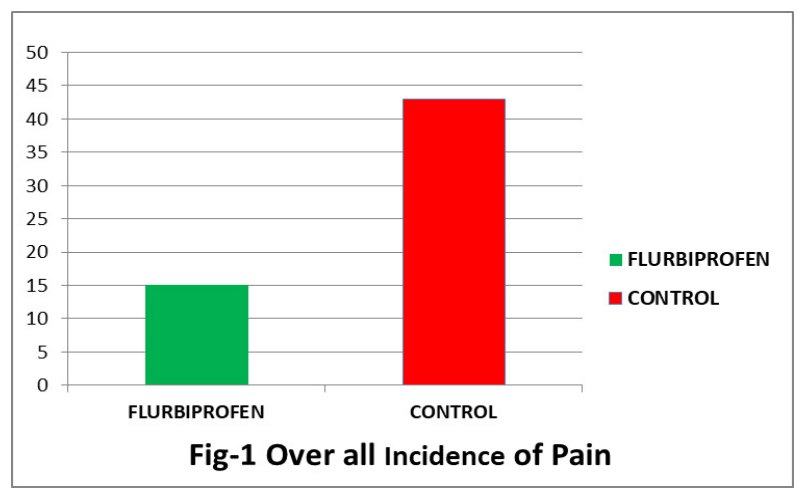

Incidence of Pain in the Flurbiprofen group is shown in green (Figure-2) and the Control group in red. At time 6h, incidence of Pain in Flurbiprofen group was $0 \%$ compared to $24 \%$ of the Control group (adjusted $\mathrm{P}$ $=.002)$; since the incidence of Pain at 6 hour in Flurbiprofen group was 0 , the Hessian matrix was singular and some convergence criteria not satisfied, an accurate $95 \%$ confidence interval (CI )or odds ratio (OR) could not be reported; at time interval 24 hours, incidence of Pain was $8 \%$ in the Flurbiprofen group compared to $34 \%$ in the Control group (adjusted $\mathrm{P}=$ .008 ) with OR 7.029 (95\% CI, 6.877-43.401); at time interval 48 hours, incidence of Pain was $9 \%$ in the Flurbiprofen group compared to $29 \%$ in the Control group (adjusted $\mathrm{P}=.034$ ) with OR 4.483 (95\% CI, 1.390- 37.490); and at time interval 72 hours, the incidence of Pain was $11 \%$ in the Flurbiprofen group compared to $24 \%$ in the Control group (adjusted $\mathrm{P}$ $=.155$ ) with OR 2.019 (95\% CI, 0.204- 30.570). Using generalized estimating equation with AR working correlation matrix and binomial probability distribution, $\mathrm{P}<.05$ was considered statistically significant.

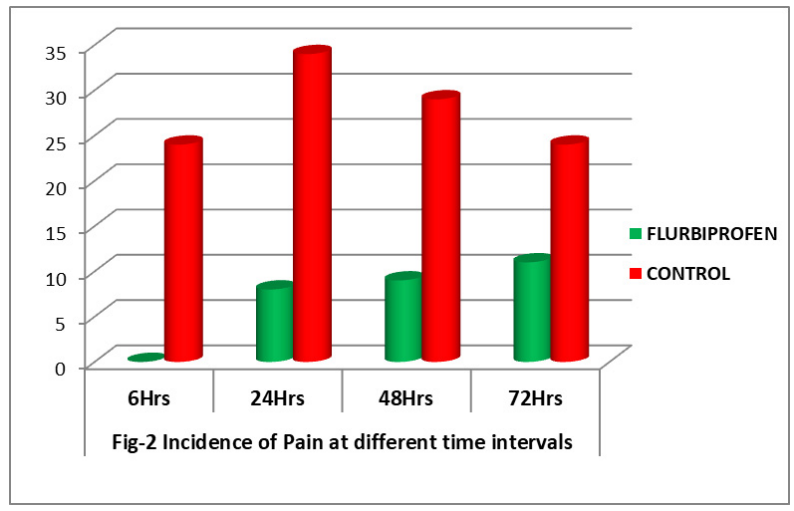

According to the VAS scores of pain (Table II) after surgery, the Flurbiprofen group exhibited significantly lower scores at all measured time points compared to the control group, indicating more efficient pain reduction. 
Table-II: Pain by VAS scale

\begin{tabular}{|l|l|l|l|}
\hline GROUP & Postoperative day 1 & Postoperative day 2 & Postoperative day 3 \\
\hline FLURBIPROFEN & $5.42 \pm 0.74$ & $4.13 \pm 1.02$ & $2.18 \pm 0.64$ \\
\hline CONTROL & $6.06 \pm 1.23$ & $4.73 \pm 1.25$ & $2.76 \pm 1.17$ \\
\hline t-value & 2.637 & 2.200 & 2.573 \\
\hline p-value & 0.010 & 0.031 & 0.012 \\
\hline
\end{tabular}

In terms of swelling degree (Table III) after surgery, even though swelling decreased in both groups from day 1 to day 3 after surgery. However, the Flurbiprofen group exhibited significantly lower swelling compared to control group at each time point measured, indicating more efficient swelling management.

Table-III Swelling degree after surgery

\begin{tabular}{|l|l|l|l|}
\hline GROUP & Postoperative day 1 & Postoperative day 2 & Postoperative day 3 \\
\hline FLURBIPROFEN & $2.18 \pm 0.26$ & $1.34 \pm 0.16$ & $0.26 \pm 0.16$ \\
\hline CONTROL & $2.85 \pm 0.43$ & $1.98 \pm 0.27$ & $1.01 \pm 0 . .37$ \\
\hline t-value & 7.888 & 12.064 & 11.007 \\
\hline p-value & $<0.0001$ & $<0.0001$ & $<0.0001$ \\
\hline
\end{tabular}

The limitation of mouth opening degree (Table IV) decreased in both groups from day 1 to day 3 after surgery. However, the Flurbiprofen group exhibited significantly lower scores than the Control group at each time point measured, indicating more efficient functional recovery after third molar surgery.

Table-IV: Limitation of mouth opening

\begin{tabular}{|l|l|l|l|}
\hline GROUP & Postoperative day 1 & Postoperative day 2 & Postoperative day 3 \\
\hline FLURBIPROFEN & $2.08 \pm 0.26$ & $1.22 \pm 0.28$ & $0.56 \pm 0.16$ \\
\hline CONTROL & $2.76 \pm 0.53$ & $1.83 \pm 0.45$ & $1.16 \pm 0.27$ \\
\hline t-value & 6.814 & 6.809 & 11.310 \\
\hline p-value & $<0.0001$ & $<0.0001$ & $<0.0001$ \\
\hline
\end{tabular}

\section{DISCUSSION}

The rate of complications in general after extraction of third molars can reach up to $20 \%$. Therefore, a key priority for dentists in these procedures is to effective prevention of these complications [10]. These symptoms include pain, dry socket, face swelling, limited mouth opening, and other, typically last for seven days causing serious impact on the daily life of patients [11]. The pathology of pain results from damaged tissues, which irritates the nerves. Edema and swelling of the face as part of the normal reactive edema after destruction of tissue during surgery. Moderate swelling is a protective reaction to trauma; however, excessive swelling has damaging consequences and even leads to infection [12]. The limited mouth opening is that cutting soft tissue during surgery irritates the temporal tendon and the muscle in nasal alar; causing a reflex spasm explains the mechanism of trismus [13]. Treatment of complications after tooth extraction include cold-hot compress, oral administration of non-steroidal anti-inflammatory drugs. These treatments need to extend for a period after surgery to shorten the durations of pain, swelling, and limited mouth opening. The inflammatory response is the most basic reaction to injuries and is a necessary stage for injury repair after third molar extraction [14].

The results of the present study showed overall incidence of $15 \%$ of Pain in group Flurbiprofen, while in Control group overall incidence of Pain was seen in $43 \%$ of the study subjects, Moreover, in our study there was a significant difference in Pain at $6 \mathrm{~h}, 24 \mathrm{~h}, 48 \mathrm{~h} \&$ 72 hour between Flurbiprofen and Control ( $p<0.05)$. In the Flurbiprofen group, Locally acting non-steroidal anti-inflammatory drugs (NSAIDs), including flurbiprofen, have been demonstrated as very effective treatments for sore throat. A potential additional benefit of formulations that are applied directly to the mucous membranes of the throat (such as lozenges, gargles, and throat sprays) is that they are known to deliver drugs locally to alleviate symptoms of pharyngitis, such as sore throat, difficulty swallowing, and swollen throat, more rapidly than systemic oral analgesia [15]. The rapid relief of symptoms is via an anti-inflammatory mechanism resulting in analgesic action. This effect is considered to be due to the local absorption which has been previously demonstrated through the buccal mucosa and ex vivo cadaveric human pharynx tissue. [16].

Our study is similar to the study by Bernard $\mathrm{P}$. Schachtel et al The dose response of flurbiprofen lozenges $(2.5,5.0$, and $12.5 \mathrm{mg}$ ) was evaluated in the treatment of sore throat. A refined version of the sore throat pain model showed that for every milligram of increase in the dose of flurbiprofen, there was an approximately 0.3 -unit increase in total pain relief $(\mathrm{P}<$ $.05)$. Flurbiprofen lozenges in all 3 dosages were well 
Tejashree R \& Hemalatha BR., Sch J Dent Sci, Jun, 2021; 8(5): 160-165

tolerated, concluded that Flurbiprofen lozenges are effective for sore throat at a dose between $5.0 \mathrm{mg}$ and $12.5 \mathrm{mg}[17]$.

Bernard Schachtel et al., studied 122 adults with acute sore throat to confirm the presence of tonsillopharyngitis and sore throat pain of at least moderate intensity ( $\geq 6$ on a $0-10$ Sore Throat Scale). Lozenges containing flurbiprofen 8.75 results showed about $78 \%$ of flurbiprofen-treated patients reported meaningful pain relief compared with $48 \%$ of placebotreated patients $(\mathrm{p}<0.01)$; Flurbiprofen lozenge was well tolerated, with no serious adverse events occurring and no patient discontinuing due to an adverse event, and demonstrated that the onset of action (time to meaningful pain relief) of flurbiprofen lozenge was $<45$ minutes [18].

A review article by Ferdinandus de Looze et al., concluded that locally administered, low-dose flurbiprofen offers a useful first-line treatment option for symptomatic relief in patients with "uncomplicated" acute pharyngitis/sore throat associated with upper respiratory tract infection, thus potentially helping to reduce unnecessary antibiotic prescribing. It also offers an effective preoperative treatment option for the reduction of early postoperative sore throat (POST) severity and incidence [19].

In these above-mentioned studies, it was suggested that the reduction in the incidence and severity of Pain, Swelling \& Trismus was related with anti-inflammatory effects of the administered drugs. Similar to these previous studies, our study found that the Flurbiprofen lozenge has reduced incidence and severity. Our study suggests that patients who received a single dose Flurbiprofen preoperatively showed decreased symptoms with increased time (in the first 6 hours) and showed reduced incidence. Nevertheless, comparisons of the effectiveness of these treatments were performed in only a low number of small studies. Studies examining the comparative effectiveness of Flurbiprofen versus other pharmacological drugs in preventing postoperative sequelae are still warranted.

\section{CONCLUSION}

In conclusion oral Flurbiprofen lozenge administered 30 minutes preoperatively can reduce complications after third molar extraction, including pain, swelling, and limited mouth opening. Besides, Flurbiprofen can promote the recovery effect, improve the quality of life after surgery, and its administration is convenient, easy, economic, and has non-invasive characteristics that deserve wider clinical attention and application.

\section{REFERENCES}

1. Gurram, P., Narayanan, V., Chandran, S., Ramakrishnan, K., Subramanian, A., \& Kalakumari, A. P. (2021). Effect of heartfulness meditation on anxiety and perceived pain in patients undergoing impacted third molar surgery. Journal of Oral and Maxillofacial Surgery.

2. Selvido, D. I., Bhattarai, B. P., Rokaya, D., Niyomtham, N., \& Wongsirichat, N. (2021). Pain in Oral and Maxillofacial Surgery and Implant Dentistry: Types and Management. European Journal of Dentistry.

3. Cho, H., Lynham, A. J., \& Hsu, E. (2017). Postoperative interventions to reduce inflammatory complications after third molar surgery: review of the current evidence. Australian dental journal, 62(4), 412-419.

4. Gulnahar, Y., \& Alpan, A. L. (2021). Comparison of postoperative morbidity between piezoelectric surgery and conventional rotary instruments in mandibular third molar surgery: a split-mouth clinical study. Medicina oral, patologia oral y cirugia bucal, 26(3), e269.

5. Cosola, S., Kim, Y. S., Park, Y. M., Giammarinaro, E., \& Covani, U. (2020). Coronectomy of Mandibular Third Molar: Four Years of Follow-Up of 130 Cases. Medicina, 56(12), 654.

6. Lambkin-Williams, R., Mann, A., \& Shephard, A. (2020). Inhibition of viral and bacterial triggerstimulated prostaglandin E2 by a throat lozenge containing flurbiprofen: An in vitro study using a human respiratory epithelial cell line. SAGE Open Medicine, 8, 2050312120960568.

7. Sheppe, A. E., \& Edelmann, M. J. (2021). Roles of eicosanoids in regulating inflammation and neutrophil migration as an innate host response to bacterial infections. Infection and Immunity, IAI00095.

8. Goncalves dos Santos, G., Li, R., Ng, M. P. E., Lemes, J. B. P., Vieira, W. F., Nagy, I., ... \& Parada, C. A. (2020). CB1 receptor- dependent desensitisation of TRPV1 channels contributes to the analgesic effect of dipyrone in sensitised primary sensory neurons. British Journal of Pharmacology, 177(20), 4615-4626.

9. Radkova, E., Burova, N., Bychkova, V., \& DeVito, R. (2017). Efficacy of flurbiprofen $8.75 \mathrm{mg}$ delivered as a spray or lozenge in patients with sore throat due to upper respiratory tract infection: a randomized, non-inferiority trial in the Russian Federation. Journal of pain research, 10, 1591.

10. Sarkar, T., \& Mandal, T. (2020). Preoperative oral zinc tablet decreases incidence of postoperative sore throat. Indian Journal of Anaesthesia, 64(5), 409.

11. Byrne, M. J., Tickle, M., Glenny, A. M., Campbell, S., Goodwin, T., \& O’Malley, L. (2019). A systematic review of quality measures used in primary care dentistry. International dental journal, 69(4), 252-264.

12. Alqooz, F., \& Al Ghatam, R. (2020). Mandibular Third Molar Postoperative Complications-A Brief Review. Journal of the Bahrain Medical Society, 32(3), 18-22. 
Tejashree R \& Hemalatha BR., Sch J Dent Sci, Jun, 2021; 8(5): 160-165

13. Tan, Y., \& Li, P. (2018). Bromelain has significant clinical benefits after extraction of the third molar during chemotherapy in patients with hematologic tumor. Oncology letters, 15(3), 2962-2966.

14. Petropolis, C., \& Antonyshyn, O. (2020). Primary Repair of Soft Tissue Injury and Soft Tissue Defects. In Facial Trauma Surgery (pp. 44-57). Content Repository Only!.

15. Burova, N., Bychkova, V., \& Shephard, A. (2018). Improvements in throat function and qualities of sore throat from locally applied flurbiprofen 8.75 $\mathrm{mg}$ in spray or lozenge format: findings from a randomized trial of patients with upper respiratory tract infection in the Russian Federation. Journal of pain research, 11, 1045.

16. Turner, R., Wevrett, S. R., Edmunds, S., Brown, M., Kulasekaran, A., Adegoke, O., \& Farrah, J. (2021). Penetration of Flurbiprofen from a Locally Applied Sore Throat Lozenge and Spray into Cadaveric Human Pharynx Tissue: A Novel ex vivo Model and Microautoradiography Method. Clinical pharmacology: advances and applications, 13, 13.

17. Schachtel, B. P., Homan, H. D., Gibb, I. A., \& Christian, J. (2002). Demonstration of dose response of flurbiprofen lozenges with the sore throat pain model. Clinical Pharmacology \& Therapeutics, 71(5), 375-380.

18. Schachtel, B., Aspley, S., Shephard, A., Schachtel, E., Lorton, M. B., \& Shea, T. (2018). Onset of analgesia by a topically administered flurbiprofen lozenge: a randomised controlled trial using the double stopwatch method. British journal of pain, 12(4), 208-216.

19. de Looze, F., Shephard, A., \& Smith, A. B. (2019). Locally delivered flurbiprofen $8.75 \mathrm{mg}$ for treatment and prevention of sore throat: a narrative review of clinical studies. Journal of pain research, 12, 3477. 